\title{
STUDY OF GLYCATED HAEMOGLOBIN AND URIC ACID LEVELS IN PROLIFERATIVE AND NON- PROLIFERATIVE DIABETIC RETINOPATHY
}

\author{
Barnali Bhattacharyya Thakur1, Deepika Lahon²
}

${ }^{1}$ Assistant Professor, Department of Biochemistry, Gauhati Medical College and Hospital, Guwahati. ${ }^{2}$ Associate Professor, Department of Biochemistry, Gauhati Medical College and Hospital, Guwahati.

\begin{abstract}
BACKGROUND

Diabetic retinopathy is one of the most common and dreadful complication of uncontrolled diabetic mellitus, which can eventually lead to blindness. Diabetic retinopathy is classified into two stages, non-proliferative and proliferative. This study was carried out to find the serum uric acid levels and glycaemic status of diabetic retinopathy cases in proliferative and nonproliferative phases. The present study was undertaken to evaluate and to assess the relationship of these factors with progress of retinopathy, both from diabetics without retinopathy to diabetic retinopathy and from non-proliferative to proliferative diabetic retinopathy. The study was carried out on 90 individuals consisting of 30 normal healthy cases as control and 60 cases of diabetes mellitus as test subjects. The test group was further subdivided into test group I comprising of controlled cases of Diabetic mellitus and test group II which included uncontrolled cases of Diabetes mellitus with retinopathy. Estimation of HbA1c was done using spectrophotometer (Spectra scan UV2600) and rest of the biochemical tests including serum uric acid was done by colorimetric principle in a colorimeter $\mathrm{HbA} 1 \mathrm{c} \%$ and serum uric acid levels were compared with controls and test group I and test group II by unpaired ' $\mathrm{t}$ ' test $\mathrm{HbA} / \mathrm{c} \%$, serum uric acid level and duration with retinopathy was assessed by chi-square test. The results were taken as significant when the probability (p) is less than 0.05 as $\%$ of the observing values of ' $t$ ' at a particular degree of freedom.

KEYWORDS
\end{abstract}

Diabetic retinopathy, Glycated Haemoglobin, Uric Acid.

HOW TO CITE THIS ARTICLE: Thakur BB, Lahon D. Study of glycated haemoglobin and uric acid levels in proliferative and nonproliferative diabetic retinopathy. J. Evolution Med. Dent. Sci. 2016;5(37):2241-2249, DOI: 10.14260/jemds/2016/521

\section{INTRODUCTION}

Diabetic retinopathy is one of the most common and dreadful complication of uncontrolled diabetic mellitus, which can eventually lead to blindness. Many factors influence the insulin secretion and insulin action in uncontrolled diabetes mellitus. Fasting blood glucose and glycated haemoglobin were related to diabetic retinopathy. ${ }^{1}$ It has been concluded that duration of diabetes, HbA1c were risk factors of diabetic retinopathy. ${ }^{2}$

Serum uric acid is known to play an important role in the insulin pattern in different types of diabetes mellitus with and without complications. Diabetic retinopathy is classified into two stages, non-proliferative and proliferative. Nonproliferative diabetic retinopathy usually appears late in the first decade or early in the second decade of the disease and is marked by retinal vascular microaneurysms, blot haemorrhage and cotton wool spots.

Proliferative retinopathy is characterized by neovascularization in response to hypoxia of the retina. Glycosylated $\mathrm{Hb}$ is formed when $\mathrm{Hb}$ combines with glucose. There are several glycated $\mathrm{Hb}$ species (HbA1a, HbA1b, HbA1c) in minor amounts in normal human blood. Of these species $\mathrm{HbA1c}$, in which glucose is linked to $\mathrm{N}$-terminal valine residues of beta chains is of the most importance as its formation is increased in diabetic persons with ambient hyperglycaemia and is used to monitor clinically for longterm control of blood suqare. ${ }^{3}$

Financial or Other, Competing Interest: None.

Submission 22-12-2015, Peer Review 16-04-2016,

Acceptance 25-04-2016, Published 09-05-2016.

Corresponding Author:

Dr. Barnali Bhattacharyya Thakur

B-201, Swapnalaya Apartment,

Gawahati-24.

E-mail: dr.barnali_gmch@yahoo.com

DOI: $10.14260 /$ jemds/2016/521

\section{AIMS AND OBJECTIVES}

1. To evaluate the serum uric acid levels and glycaemic status of diabetic retinopathy cases in proliferative and non-proliferative phases.

2. To assess the relationship of the above factors with the progress of retinopathy from uncomplicated diabetic cases to diabetic retinopathy and from non-proliferative diabetic retinopathy to proliferative diabetic retinopathy.

3. To utilize the data for a new clinical approach for better management of Diabetic retinopathy.

\section{Scheme of Study}

1. The Study was carried out in outpatient and inpatient patients of the Department of Ophthalmology, Guwahati Medical College and Hospital, Guwahati-32, during the period of one year from October 2014 to October 2015.

2. Cases of proliferative and non-proliferative diabetic retinopathy were included in the study and equal number of non-diabetics and diabetics without retinopathy with age and sex matched were taken as control.

3. Evaluation of uric acid level and glycosylated $\mathrm{Hb}$ were done in subjects of both groups.

4. Statistical evaluation of validity of the obtained results.

5. To formulate a pattern of biochemical parameters under the study conditions.

6. To make an integrated analysis of the results for evaluating the utilization index under the proposed study.

\section{MATERIALS AND METHODS}

1. To estimate uric acid by Uricase/PAP method with uric acid kit of Crest Biosystems.

2. To estimate glycosylated $\mathrm{Hb}$ with standard glycosylated $\mathrm{Hb}$ kit utilizing cation exchange resin method was used. 


\section{RESULTS AND OBSERVATIONS}

In the present study 30 cases each of Diabetes mellitus without retinopathy (Test Group I) and Diabetes mellitus with retinopathy (Test Group II) were selected at random from the outpatient department and admitted cases of the Department of Ophthalmology, Guwahati Medical College. The study was conducted from October 2014-October 2015. The control group consisted of 30 normal healthy subjects with age and sex evenly matched.

\section{A. Age}

The age of both the controls and test groups are shown in Table IA. The control group consisted of 30 healthy individuals with age ranging from 30 to 80 years with mean value of 53.241, standard deviation of 10.38. Most of the healthy subjects were in 50-59 years age group with relative frequency of 0.366 (Table - IB). The test Group I consisted of 30 patients with diabetes mellitus without retinopathy with age ranging from 30-80 years with mean value of 52.53 and standard deviation of 10.85 . Most of the patients were in age group of 50-59 years with relative frequency of 0.33 (Table

IB). The test Group II consisted of 30 patients of diabetes mellitus with retinopathy and age ranging from 30-80 years with mean value of 55.53 and standard deviation of 7.84 . Maximum patients were in age group of 50-59 years with relative frequency of 0.5 (Table - IB).

\section{Sex}

The sex of the control and test groups were shown in Table 1A. The sex distribution of control and test groups are shown in Table $1 \mathrm{C}$.

In the Healthy control group there were 30 individuals comprising of 18 males and 12 females, i.e. $60 \%$ were male and $40 \%$ were female as shown in (Table $1 \mathrm{C}$ ).

In test Group I there were 30 individuals with 17 males and 13 females, i.e. $56.7 \%$ were males and $43.3 \%$ females. (Table 1C) The test Group II comprised of 30 individuals with 18 males and 12 females with a distribution of $60 \%$ and $40 \%$ respectively as shown in Table $1 \mathrm{C}$.

The fasting serum glucose levels expressed in $\mathrm{mg} / \mathrm{dL}$ of the controls and the test subjects are shown in Table No. ID. The mean fasting serum glucose levels and other statistical parameters are shown in Figure No. II e and Table ID.

The control group comprising of 30 individuals had a mean fasting serum glucose of $76.666 \pm 7.18 \mathrm{mg} / \mathrm{dL}$, ranging from $65-89 \mathrm{mg} / \mathrm{dL}$ and a median of $77.5 \mathrm{mg} / \mathrm{dL}$.

The test group which was further divided into 2 subcategories Group I and Group II, had a mean fasting serum glucose value of $97.966 \pm 12.59$ and $238.93 \pm 73.86 \mathrm{mg} / \mathrm{dL}$ and a median value of 94 and $260 \mathrm{mg} / \mathrm{dL}$ respectively.

Comparison of fasting serum glucose levels between the controls and the different categories of test subjects revealed statistically significant difference between controls and test Group I and test Group II.

The difference of the mean fasting serum glucose values between healthy control and test Group I is highly significant with a ' $\mathrm{t}$ ' value of 8.049 and corresponding $\mathrm{P}<0.0001$. The difference of mean fasting glucose between the healthy control and test Group II is highly significant with a ' $t$ ' value of 11.977 and corresponding $\mathrm{P}<0.0001$. The difference of mean fasting glucose between test Group I and test Group II is also highly significant with a ' $t$ ' value of 10.305 and corresponding $\mathrm{P}<0.0001$.

The HbA1c levels, expressed in \%, of the controls and the test subjects are shown in Table No. 1G. The mean HbA1c levels and other statistical parameters are shown in Figure II $\mathrm{f}$ and Table No. 1G. The control group comprising of 30 individuals had a mean $\mathrm{HbA} 1 \mathrm{c}$ level of $4.90 \pm 0.813 \%$ ranging from $3.8-6.5 \%$ and a median value of $5 \%$. The test Group I and test Group II had a mean HbA1c value of $5.03 \pm 1.060$ and $10.734 \pm 2.810 \%$ and median value of $5.11 \%$ and $11.05 \%$ respectively.

Comparison of HbA1c levels between controls and test Group I and test Group II revealed statistically significant difference.

The difference of the mean HbA1c values between healthy control and test Group I does not reveal any significance with a ' $\mathrm{t}$ ' value of 0.533 and corresponding $\mathrm{P}=0.596$. The difference of mean $\mathrm{HbA1c}$ values between healthy controls and test Group II is highly significant with a ' $t$ ' value of 10.924 and corresponding $\mathrm{P}<0.0001$.

The difference of mean HbA1c values between test Group I and test Group II is significant with ' $t$ ' value of 10.403 and corresponding $\mathrm{P}<0.0001$.

The serum uric acid levels expressed in $\mathrm{mg} / \mathrm{dL}$ of the controls and test subjects are shown in Table No. $1 \mathrm{I}$.

The mean serum uric acid levels and other statistical parameters are shown in Figure No. II g and Table No $1 \mathrm{~J}$.

The control group comprising of 30 individuals had a mean serum uric acid level of $4.136 \pm 0.901 \mathrm{mg} / \mathrm{dL}$, ranging from $2.5-6.1 \mathrm{mg} / \mathrm{dL}$ and a median value of $4 \mathrm{mg} / \mathrm{dL}$.

The test group, which was further divided into 2 subcategories Group I and Group II had a mean serum uric acid value of $4.346 \pm 0.942 \mathrm{mg} / \mathrm{dL}$ and $2.99 \pm 0.605 \mathrm{mg} / \mathrm{dL}$ and median value of 4 and $3 \mathrm{mg} / \mathrm{dL}$ respectively.

Comparison of serum uric acid levels between controls and test Group II and test Group I and test Group II revealed statistically significant difference indicating serum uric acid levels are lowered in patients with diabetic retinopathy. The difference of the mean serum uric acid values between control and test Group I does not reveal any significance with a ' $t$ ' value of 0.882 and corresponding $\mathrm{P}=0.381$.

The difference of mean serum uric acid values between control and test Group II is highly significant with ' $\mathrm{t}$ ' -5.784 and corresponding $\mathrm{P}<0.0001$. The difference of mean serum uric acid level between test Group I and test Group II is significant with ' $\mathrm{t}$ ' value of -6.634 and corresponding $\mathrm{P}<0.0001$.

Table - $\mathrm{K}$ shows level of $\mathrm{HbA} 1 \mathrm{c} \%$ and type of Retinopathy. Majority of patients presenting with nonproliferative retinopathy had $\mathrm{HbA} 1 \mathrm{c} \%$ less than $9 \%$. It is clear that majority of patients who had proliferative retinopathy has $\mathrm{HbA} 1 \mathrm{c} \%$ above $11 \%$.

Table: $\mathrm{L}$ shows level of serum uric acid in $\mathrm{mg} \%$ and type of Retinopathy. Majority of patients presenting with nonproliferative retinopathy had serum uric acid level less then 4 $\mathrm{mg} / \mathrm{dL}$. It is also observed that most patients with proliferative retinopathy had serum uric acid levels also less than $4 \mathrm{mg} / \mathrm{dL}$.

Correlation between glycated $\mathrm{Hb}$ and Uric acid in Group II shows no significant correlation with $\mathrm{P}=.2937$, (Fig. II h). 
Correlation between glycated $\mathrm{Hb}$ and Uric acid in nonproliferative stage shows no significant correlation with $\mathrm{P}=.9654$, (Fig. II i).

Correlation between glycated $\mathrm{Hb}$ and Uric acid in proliferative stage shows $p=0.0371$, which is significant (Fig. II j).

Shows the relation between $\mathrm{HbA1c} \%$ level and duration with the type of retinopathy. Majority of patients presenting with non-proliferative retinopathy had $\mathrm{HbA} 1 \mathrm{c} \%$ between 7 $9 \%$. The chi-square value being 22.94 and $\mathrm{P}$ value $<0.0001$, which is highly significant. Majority of patients with proliferative retinopathy had $\mathrm{HbA1c} \%$ above $11 \%$.
Majority of patients with non-proliferative retinopathy had duration of diabetes mellitus less than 10 years and most of the patients with proliferative retinopathy had a duration of more than 10 years. The chi-square value being 16.101 and $\mathrm{P}=0.0001$, which is statistically significant.

Shows relationship of uric acid level with retinopathy. Majority of patients presenting with non-proliferative retinopathy had uric acid level less than 4 . It is also observed that majority of patients with proliferative retinopathy had uric acid level between $2-4 \mathrm{mg} / \mathrm{dL}$. The chi-square value being 0.040 and $\mathrm{P}=0.8003$, which is not statistically significant.

\begin{tabular}{|c|c|c|c|c|c|c|c|c|}
\hline \multicolumn{3}{|c|}{ Control Group } & \multicolumn{3}{|c|}{$\begin{array}{c}\text { Test Group-I } \\
\text { (Diabetes Mellitus Without Retinopathy) }\end{array}$} & \multicolumn{3}{|c|}{$\begin{array}{c}\text { Test Group-II } \\
\text { (Diabetes Mellitus Without Retinopathy) }\end{array}$} \\
\hline Sl. No. & Age & Sex & Sl. No. & Age & Sex & Sl. No. & Age & Sex \\
\hline 1 & 34 & $\mathrm{M}$ & 1 & 55 & $\mathrm{M}$ & 1 & 57 & $\mathrm{M}$ \\
\hline 2 & 50 & M & 2 & 60 & $\mathrm{~F}$ & 2 & 45 & $\mathrm{M}$ \\
\hline 3 & 52 & $\mathrm{M}$ & 3 & 55 & $F$ & 3 & 50 & $\mathrm{~F}$ \\
\hline 4 & 62 & $\mathrm{~F}$ & 4 & 45 & $\mathrm{~F}$ & 4 & 43 & $\mathrm{M}$ \\
\hline 5 & 65 & $\mathrm{~F}$ & 5 & 48 & $\mathrm{M}$ & 5 & 61 & $\mathrm{~F}$ \\
\hline 6 & 44 & $M$ & 6 & 43 & $\mathrm{M}$ & 6 & 56 & $\mathrm{~F}$ \\
\hline 7 & 48 & $\mathrm{M}$ & 7 & 48 & $\mathrm{M}$ & 7 & 55 & $\mathrm{~F}$ \\
\hline 8 & 62 & $\mathrm{M}$ & 8 & 58 & $\mathrm{~F}$ & 8 & 50 & $\mathrm{M}$ \\
\hline 9 & 58 & $\mathrm{M}$ & 9 & 60 & $\mathrm{~F}$ & 9 & 39 & $\mathrm{M}$ \\
\hline 10 & 66 & $\mathrm{~F}$ & 10 & 53 & $\mathrm{~F}$ & 10 & 60 & $\mathrm{M}$ \\
\hline 11 & 46 & M & 11 & 43 & $\mathrm{M}$ & 11 & 58 & $\mathrm{~F}$ \\
\hline 12 & 35 & $\mathrm{~F}$ & 12 & 62 & $\mathrm{~F}$ & 12 & 40 & $\mathrm{M}$ \\
\hline 13 & 56 & $\mathrm{M}$ & 13 & 60 & $\mathrm{M}$ & 13 & 65 & $\mathrm{~F}$ \\
\hline 14 & 45 & $\mathrm{M}$ & 14 & 59 & M & 14 & 49 & $\mathrm{M}$ \\
\hline 15 & 44 & $\mathrm{~F}$ & 15 & 65 & $\mathrm{M}$ & 15 & 58 & $\mathrm{M}$ \\
\hline 16 & 75 & $\mathrm{~F}$ & 16 & 30 & $\mathrm{M}$ & 16 & 60 & $\mathrm{M}$ \\
\hline 17 & 32 & $\mathrm{~F}$ & 17 & 49 & $\mathrm{~F}$ & 17 & 58 & $\mathrm{M}$ \\
\hline 18 & 55 & $\mathrm{~F}$ & 18 & 72 & $\mathrm{M}$ & 18 & 60 & $\mathrm{~F}$ \\
\hline 19 & 63 & $\mathrm{M}$ & 19 & 58 & $\mathrm{~F}$ & 19 & 59 & $\mathrm{M}$ \\
\hline 20 & 59 & $\mathrm{M}$ & 20 & 60 & $\mathrm{~F}$ & 20 & 56 & $\mathrm{~F}$ \\
\hline 21 & 54 & $\mathrm{M}$ & 21 & 58 & $\mathrm{M}$ & 21 & 52 & $\mathrm{M}$ \\
\hline 22 & 54 & $\mathrm{M}$ & 22 & 52 & $M$ & 22 & 70 & $\mathrm{M}$ \\
\hline 23 & 48 & $\mathrm{~F}$ & 23 & 48 & M & 23 & 50 & $\mathrm{~F}$ \\
\hline 24 & 64 & $\mathrm{~F}$ & 24 & 30 & $\mathrm{M}$ & 24 & 55 & $\mathrm{~F}$ \\
\hline 25 & 59 & $\mathrm{M}$ & 25 & 40 & $\mathrm{M}$ & 25 & 53 & $\mathrm{~F}$ \\
\hline 26 & 56 & $\mathrm{M}$ & 26 & 30 & $\mathrm{~F}$ & 26 & 62 & $\mathrm{M}$ \\
\hline 27 & 44 & $\mathrm{~F}$ & 27 & 70 & $M$ & 27 & 70 & $\mathrm{M}$ \\
\hline 28 & 46 & $\mathrm{M}$ & 28 & 58 & $\mathrm{~F}$ & 28 & 66 & $\mathrm{M}$ \\
\hline 29 & 68 & $\mathrm{M}$ & 29 & 60 & $\mathrm{M}$ & 29 & 60 & $\mathrm{~F}$ \\
\hline 30 & 50 & $\mathrm{~F}$ & 30 & 45 & $\mathrm{f}$ & 30 & 59 & $\mathrm{M}$ \\
\hline
\end{tabular}

\begin{tabular}{|c|c|c|c|c|}
\hline Category & Range (Yrs.) & Frequency & Relatives Frequency & Mean (Yrs.) \\
\hline Control & $\begin{array}{l}30-39 \\
40-49 \\
50-59 \\
60-69 \\
70-79\end{array}$ & $\begin{array}{c}3 \\
8 \\
11 \\
7 \\
1\end{array}$ & $\begin{array}{c}0.1 \\
0.266 \\
0.366 \\
0.233 \\
0.03\end{array}$ & 53.241 \\
\hline $\begin{array}{c}\text { Test Group-I } \\
\text { (Diabetes Mellitus without Retinopathy) }\end{array}$ & $\begin{array}{l}30-39 \\
40-49 \\
50-59 \\
60-69 \\
70-79 \\
\end{array}$ & $\begin{array}{c}3 \\
8 \\
10 \\
7 \\
2 \\
\end{array}$ & $\begin{array}{c}0.1 \\
0.266 \\
0.33 \\
0.23 \\
0.06 \\
\end{array}$ & 52.466 \\
\hline $\begin{array}{c}\text { Test Group-II } \\
\text { (Diabetes Mellitus with Retinopathy) }\end{array}$ & $\begin{array}{l}30-39 \\
40-49 \\
50-59 \\
60-69 \\
70-79\end{array}$ & $\begin{array}{c}1 \\
4 \\
15 \\
8 \\
2\end{array}$ & $\begin{array}{c}0.03 \\
0.133 \\
0.5 \\
0.266 \\
0.06\end{array}$ & 55.53 \\
\hline
\end{tabular}


Frequency Distribution Study of Subject with Age in Different Groups

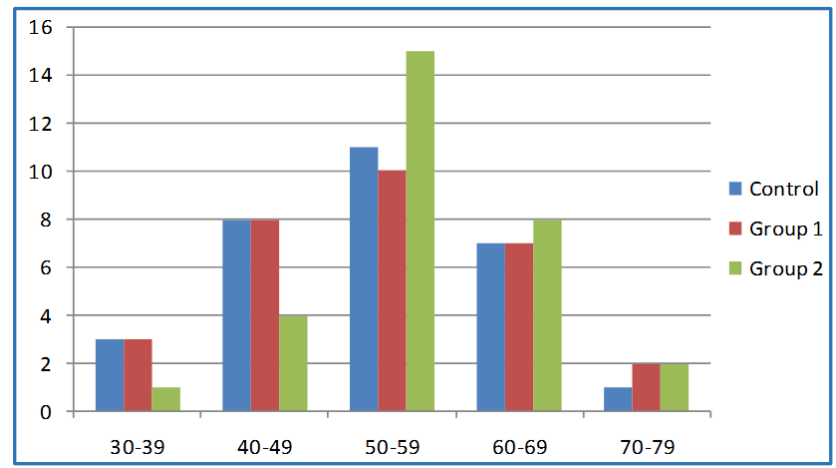

Range of Age (Years) in Different Groups

Fig. II a: Frequency Distribution Study of Subject with Age in Different Groups

\begin{tabular}{|c|c|c|c|c|c|c|}
\hline \multirow{2}{*}{ Sex } & \multicolumn{2}{|c|}{ Control } & \multicolumn{2}{c|}{ Test Group-I } & Test Group-II \\
\cline { 2 - 7 } & No. & $\begin{array}{c}\text { Distribu } \\
\text { tion }\end{array}$ & $\begin{array}{c}\text { No } \\
.\end{array}$ & $\begin{array}{c}\text { Distrib } \\
\text { ution }\end{array}$ & No. & $\begin{array}{c}\text { Distribut } \\
\text { ion }\end{array}$ \\
\hline $\mathrm{M}$ & 18 & $60 \%$ & 17 & $56.7 \%$ & 18 & $60 \%$ \\
\hline $\mathrm{F}$ & 12 & $40 \%$ & 13 & $43.3 \%$ & 12 & $40 \%$ \\
\hline Total & $\mathbf{3 0}$ & $\mathbf{1 0 0 \%}$ & $\mathbf{3 0}$ & $\mathbf{1 0 0 \%}$ & & $\mathbf{1 0 0 \%}$ \\
\hline \multicolumn{7}{|c|}{$\begin{array}{r}\text { Table 1C: Showing \% Distribution of Sex } \\
\text { in Healthy Control and Test Groups }\end{array}$} \\
\hline
\end{tabular}

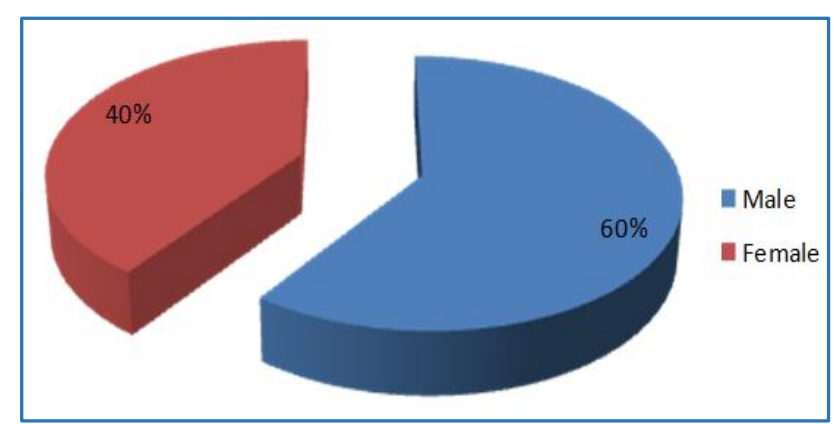

Fig. II b: Showing Male and Female Ratio in Control Group

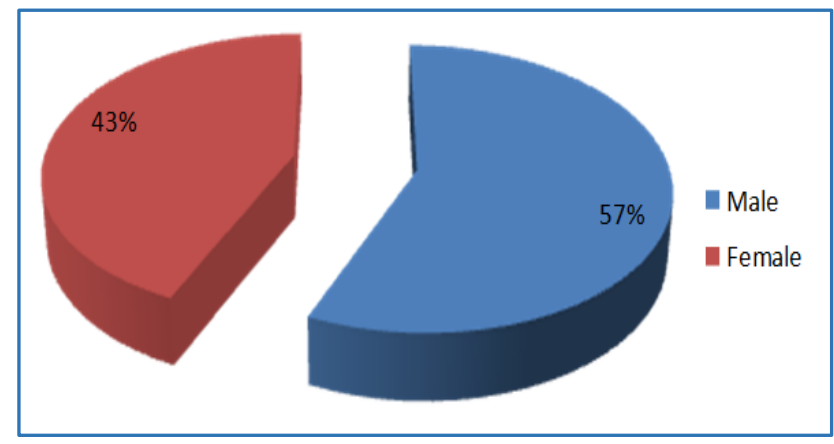

Fig. II c: Showing Male and Female Ratio in Test Group I

Test Group II

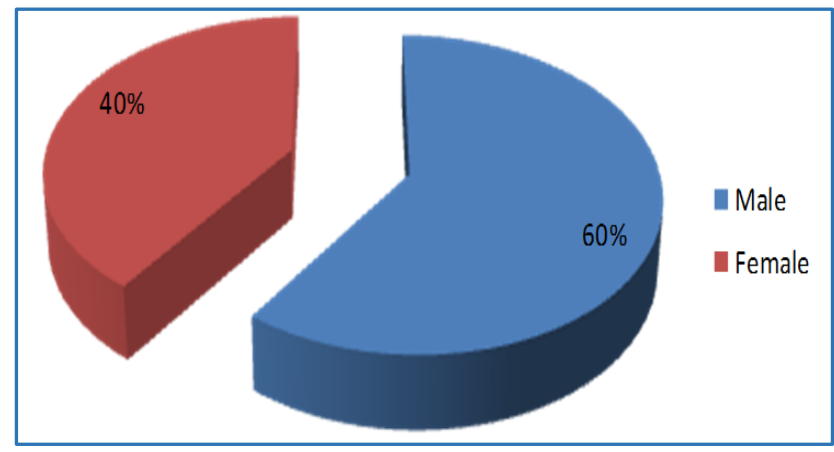

Fig. II d: Showing Male and Female Ratio in Test Group II

\begin{tabular}{|c|c|c|c|c|c|c|c|c|c|}
\hline \multicolumn{4}{|c|}{ Control } & \multicolumn{3}{|c|}{ Test Group-I } & \multicolumn{3}{|c|}{ Test Group-II } \\
\hline $\begin{array}{l}\text { Sl. } \\
\text { No. }\end{array}$ & $\begin{array}{c}\text { Fasting } \\
\text { Glucose } \\
\text { mg/dL }\end{array}$ & $\begin{array}{c}\text { HbA1c } \\
\%\end{array}$ & $\begin{array}{c}\text { Sr. uric } \\
\text { Acid } \\
\text { mg/dL }\end{array}$ & $\begin{array}{c}\text { Fasting } \\
\text { Glucose } \\
\text { mg/dL }\end{array}$ & $\begin{array}{c}\text { HbA1c } \\
\%\end{array}$ & $\begin{array}{c}\text { Sr. uric } \\
\text { Acid } \\
\text { mg/dL }\end{array}$ & $\begin{array}{c}\text { Fasting } \\
\text { Glucose } \\
\text { mg/dL }\end{array}$ & $\begin{array}{c}\text { HbA1c. } \\
\%\end{array}$ & $\begin{array}{c}\text { Sr. uric } \\
\text { Acid } \\
\text { mg/dL }\end{array}$ \\
\hline 1 & 84 & 6.5 & 2.5 & 126 & 5.31 & 4 & 130 & 7.26 & 2.5 \\
\hline 2 & 70 & 4 & 3 & 100 & 7 & 5 & 140 & 14.7 & 4 \\
\hline 3 & 79 & 5 & 4 & 90 & 6.05 & 3 & 260 & 14 & 3.2 \\
\hline 4 & 77 & 6 & 4.2 & 110 & 5.13 & 3.6 & 200 & 13.5 & 2 \\
\hline 5 & 88 & 6.1 & 3.5 & 92 & 4 & 2.8 & 170 & 8.48 & 2.1 \\
\hline 6 & 89 & 5.5 & 3.1 & 87 & 4 & 4 & 300 & 11.2 & 2.0 \\
\hline 7 & 65 & 4 & 2.8 & 89 & 4.6 & 4 & 201 & 11.5 & 2.5 \\
\hline 8 & 78 & 4.5 & 4 & 101 & 4.1 & 2.8 & 374 & 11.3 & 3 \\
\hline 9 & 66 & 3.8 & 4.5 & 120 & 3.15 & 3.6 & 198 & 8 & 2.9 \\
\hline 10 & 75 & 3.5 & 3.8 & 96 & 5.2 & 3.5 & 200 & 8.8 & 4 \\
\hline 11 & 72 & 4.5 & 3.5 & 89 & 6 & 4 & 176 & 9.4 & 3.8 \\
\hline 12 & 70 & 4 & 4 & 92 & 4.5 & 5.1 & 287 & 12.6 & 3.2 \\
\hline 13 & 70 & 5.5 & 2.5 & 90 & 4.2 & 5 & 140 & 14.9 & 3 \\
\hline 14 & 83 & 5.13 & 3 & 100 & 6.6 & 6.1 & 143 & 13 & 3 \\
\hline 15 & 68 & 5.2 & 4.8 & 89 & 6 & 5.2 & 120 & 13.1 & 3.5 \\
\hline 16 & 84 & 5.13 & 4 & 87 & 5 & 4 & 198 & 12 & 3.2 \\
\hline
\end{tabular}




\begin{tabular}{|c|c|c|c|c|c|c|c|c|c|}
\hline 17 & 88 & 4.9 & 4 & 110 & 4.5 & 3.8 & 276 & 12.9 & 4 \\
\hline 18 & 79 & 5.13 & 5 & 100 & 5.2 & 3 & 280 & 9.4 & 2.5 \\
\hline 19 & 70 & 3.8 & 5.1 & 126 & 5.2 & 3.7 & 261 & 10.9 & 2.0 \\
\hline 20 & 79 & 4 & 4.5 & 120 & 3.16 & 3.6 & 261 & 9.9 & 2.1 \\
\hline 21 & 77 & 6.4 & 4.8 & 110 & 3.5 & 4 & 400 & 14.9 & 3 \\
\hline 22 & 70 & 4.4 & 5.1 & 100 & 6.2 & 4.8 & 276 & 7.8 & 3.2 \\
\hline 23 & 66 & 5.13 & 5.6 & 90 & 6.1 & 5.2 & 200 & 6.8 & 3 \\
\hline 24 & 84 & 5.59 & 5 & 87 & 5.5 & 5 & 375 & 6 & 3 \\
\hline 25 & 83 & 5 & 5 & 89 & 3.8 & 5 & 300 & 5.5 & 2.8 \\
\hline 26 & 80 & 5 & 4 & 96 & 6 & 6 & 280 & 11.5 & 3 \\
\hline 27 & 72 & 4.9 & 4.8 & 77 & 4 & 5.1 & 276 & 8.7 & 2.9 \\
\hline 28 & 80 & 5.8 & 3.9 & 87 & 5.1 & 6 & 287 & 8 \\
\hline 29 & 85 & 6.0 & 6.1 & 89 & 5 & 4.5 & 260 & 15.2 & 3.4 \\
\hline 30 & 70 & 3.0 & 4 & 100 & 6.8 & 5 & 200 & 10.8 & 4 \\
\hline \multicolumn{7}{|c|}{ Table 1D: Serum Glucose, HbA1c and Uric Acid Levels in Control, Test Group-I and Test Group-II } \\
\hline
\end{tabular}

\begin{tabular}{|c|c|c|c|c|c|c|c|}
\hline \multicolumn{2}{|c|}{ Fasting Serum Glucose in mg/dL } \\
\hline Category & Nos. of Case & Mean & S.D. & Range & $\begin{array}{c}\text { Coefficient of } \\
\text { Variation }\end{array}$ & Median & SEM \\
\hline Control & 30 & 76.666 & \pm 7.18 & $65-89$ & 9.36 & 77.5 & 1.3116 \\
\hline Test Group I & 30 & 97.966 & \pm 12.59 & $17-126$ & 12.85 & 94 & 2.2992 \\
\hline Test Group II & 30 & 238.93 & \pm 73.86 & $120-400$ & 30.9 & 260 & 13.4856 \\
\hline \multicolumn{7}{|c|}{ Table No. 1E: Fasting Serum Glucose Levels in Controls and Test Subjects } \\
\hline
\end{tabular}

\begin{tabular}{|c|c|c|c|c|c|c|c|c|c|}
\hline \multirow[t]{2}{*}{ Parameter } & \multicolumn{3}{|c|}{ Control V/S Test Group-I } & \multicolumn{3}{|c|}{ Control V/S Test Group-II } & \multicolumn{3}{|c|}{$\begin{array}{c}\text { Test Group-I } \\
\text { V/S Test Group-II }\end{array}$} \\
\hline & Df & 't' & P value & Df & 't' & P value & Df & 't' & P value \\
\hline $\begin{array}{l}\text { Fasting Serum } \\
\text { Glucose }\end{array}$ & 58 & 8.049 & $<0.0001$ & 58 & 11.977 & $<0.0001$ & 58 & 10.305 & $<0.0001$ \\
\hline
\end{tabular}

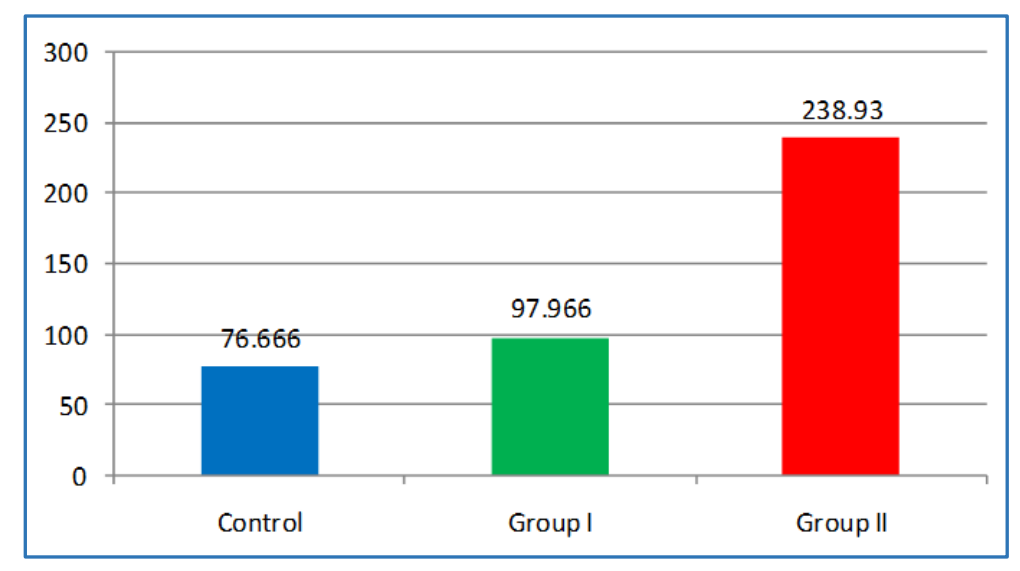

Fig. II E: Mean FBS Levels in different Groups

\begin{tabular}{|c|c|c|c|c|c|c|c|}
\hline \multicolumn{8}{|c|}{ HbA1c Levels in \% } \\
\hline Category & $\begin{array}{c}\text { No. of } \\
\text { Case }\end{array}$ & Mean & S.D. & Range & $\begin{array}{c}\text { Coefficient of } \\
\text { Variation }\end{array}$ & Median & SEM \\
\hline Control & 30 & 4.90 & \pm 0.813 & $3.8-6.5$ & 16.59 & 5.0 & 0.1485 \\
\hline Test Group I & 30 & 5.03 & \pm 1.060 & $3.15-7$ & 12.07 & 5.11 & 0.1937 \\
\hline Test Group II & 30 & 10.734 & \pm 2.810 & $6-15.2$ & 26.178 & 11.05 & 0.1531 \\
\hline \multicolumn{7}{|l}{ Table 1G: HbA1c (\%) Levels in Controls and Test Subjects } \\
\hline
\end{tabular}

\begin{tabular}{|c|c|c|c|c|c|c|c|c|c|}
\hline \multirow[t]{2}{*}{ Parameter } & \multicolumn{3}{|c|}{$\begin{array}{l}\text { Control V/S Test } \\
\text { Group-I }\end{array}$} & \multicolumn{3}{|c|}{ Control V/S Test Group-II } & \multicolumn{3}{|c|}{$\begin{array}{l}\text { Test Group-I } \\
\text { V/S Test Group-II }\end{array}$} \\
\hline & Df & 't' & P value & Df & 't' & P value & Df & 't' & P value \\
\hline HbA1c (\%) & 58 & 0.533 & 0.5961 & 58 & 10.924 & $<0.0001$ & 58 & 10.403 & $<0.0001$ \\
\hline
\end{tabular}




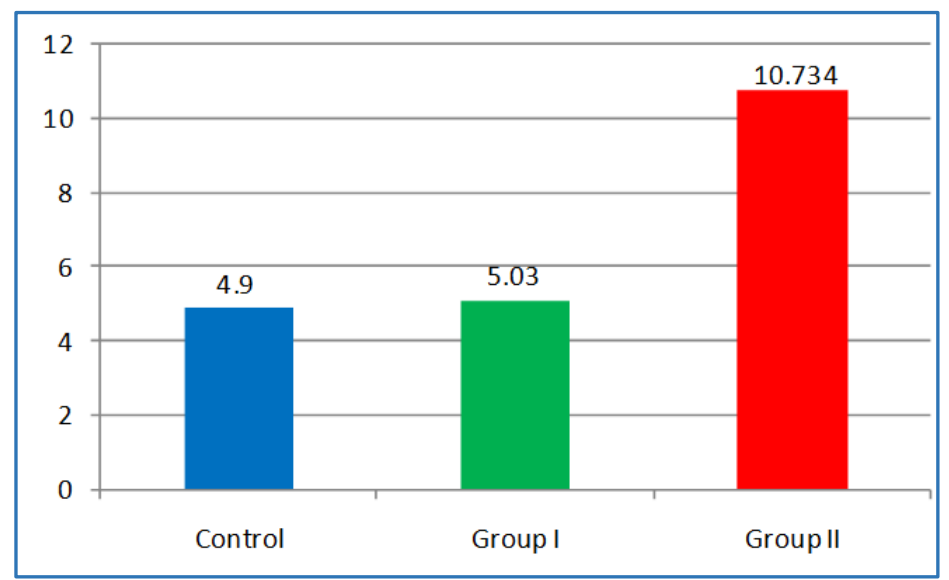

Fig. II f: Mean HbA1c Levels in Different Groups

Different Groups

Serum Uric Acid Levels in Controls and Test Subjects

\begin{tabular}{|c|c|c|c|c|c|c|c|}
\hline \multicolumn{7}{|c|}{ HbA1c Levels in \% } \\
\hline Category & No. of Cases & Mean & S.D. & Range & Coefficient of Variation & Median & SEM \\
\hline Control & 30 & 4.136 & \pm 0.901 & $2.5-6.1$ & 21.78 & 4 & 0.1647 \\
\hline Test Group I & 30 & 4.346 & \pm 0.942 & $2.8-6.1$ & 21.67 & 4 & 0.1720 \\
\hline Test Group II & 30 & 2.99 & \pm 0.605 & $2-4$ & 20.234 & 3 & 0.1106 \\
\hline \multicolumn{7}{|c|}{ Table 1 (I): Serum Uric Acid in $\mathbf{~ m g / d L}$} \\
\hline
\end{tabular}

\begin{tabular}{|c|c|c|c|c|c|c|c|c|c|}
\hline \multirow[t]{2}{*}{ Parameter } & \multicolumn{3}{|c|}{ Control V/S Test Group-I } & \multicolumn{3}{|c|}{ Control V/S Test Group-II } & \multicolumn{3}{|c|}{ Test Group-I V/S Test Group-II } \\
\hline & Df & 't' & P value & Df & 't' & P value & Df & 't' & P value \\
\hline Uric Acid & 58 & 0.882 & 0.381 & 58 & -5.784 & $<0.0001$ & 58 & -6.634 & $<0.0001$ \\
\hline
\end{tabular}

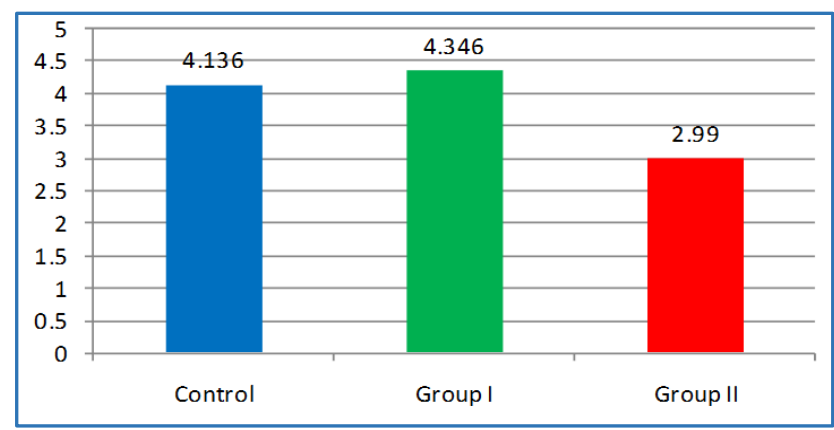

Fig. IIg: Mean Uric Acid Levels in Different Groups

\begin{tabular}{|c|c|c|}
\hline \multirow{2}{*}{ Hba1C\% } & \multicolumn{2}{|c|}{ No. of Patients } \\
\cline { 2 - 3 } & Non-proliferative & Proliferative \\
\hline$<7$ & 3 & --- \\
\hline $7-9$ & 8 & --- \\
\hline $9-11$ & 4 & -- \\
\hline $11+$ & 2 & 13 \\
\hline Total & 17 & 13 \\
\hline Table 1L: HbA1c\% Level and Type of Retinopathy \\
\hline
\end{tabular}

\begin{tabular}{|c|c|c|}
\hline \multirow{2}{*}{$\begin{array}{c}\text { Uric Acid } \\
\text { mg/dL }\end{array}$} & \multicolumn{2}{|c|}{ No. of Patients } \\
\cline { 2 - 3 } & Non-proliferative & Proliferative \\
\hline$<2$ & --- & --- \\
\hline $2-4$ & 14 & 12 \\
\hline $4-6$ & 3 & 1 \\
\hline $6+$ & --- & --- \\
\hline Total & 17 & 13 \\
\hline \multicolumn{2}{|c|}{ Table 1M: Uric Acid mg/dL Level and Type of } \\
Retinopathy
\end{tabular}

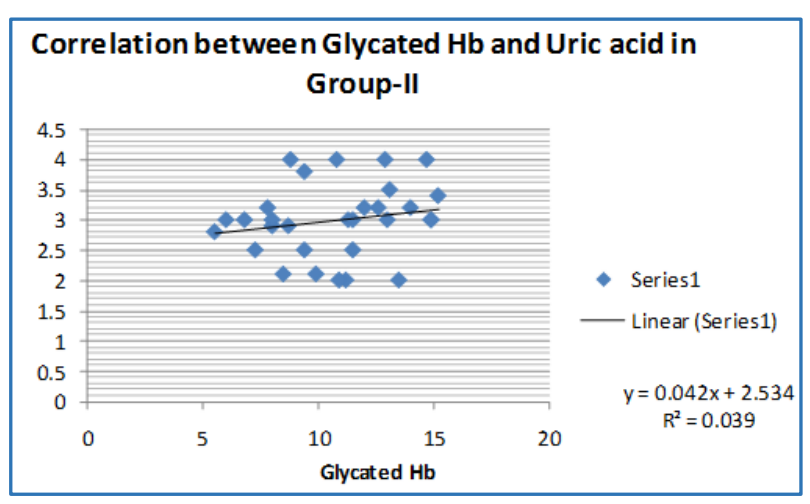

Fig. II h: Correlation between HbA1c\% and Serum Uric Acid in Patients with Diabetic Retinopathy in Test Group-II

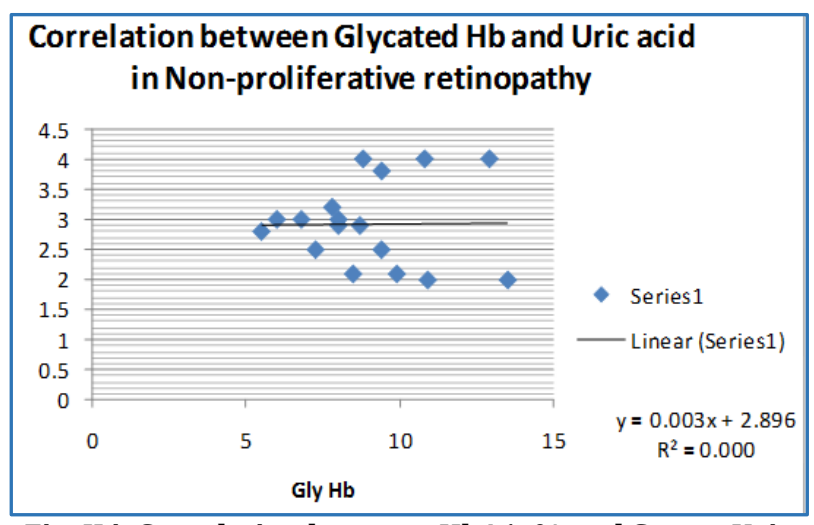

Fig. II i: Correlation between HbA1c\% and Serum Uric Acid in Patients with Non-Proliferative Diabetic Retinopathy 


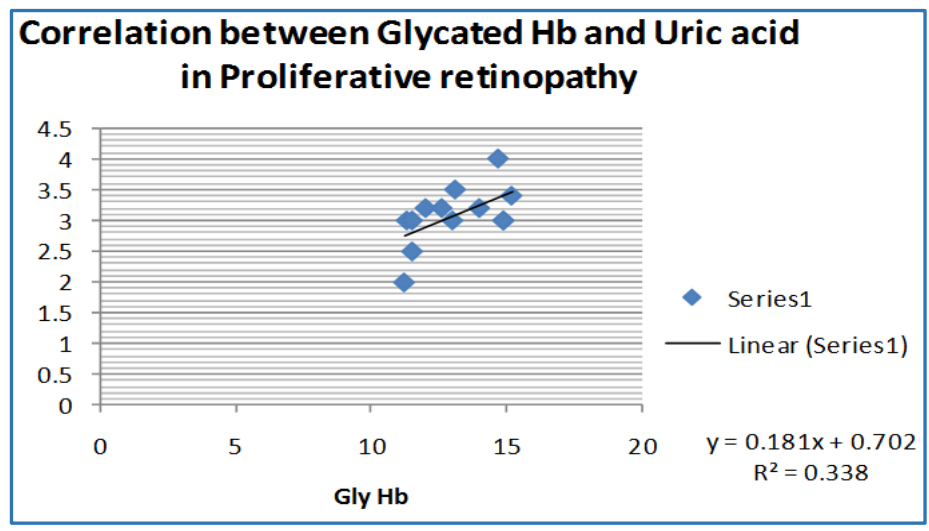

Fig. II j: Correlation of HbA1c\% and Serum Uric Acid in Patients with Proliferative Diabetic Retinopathy

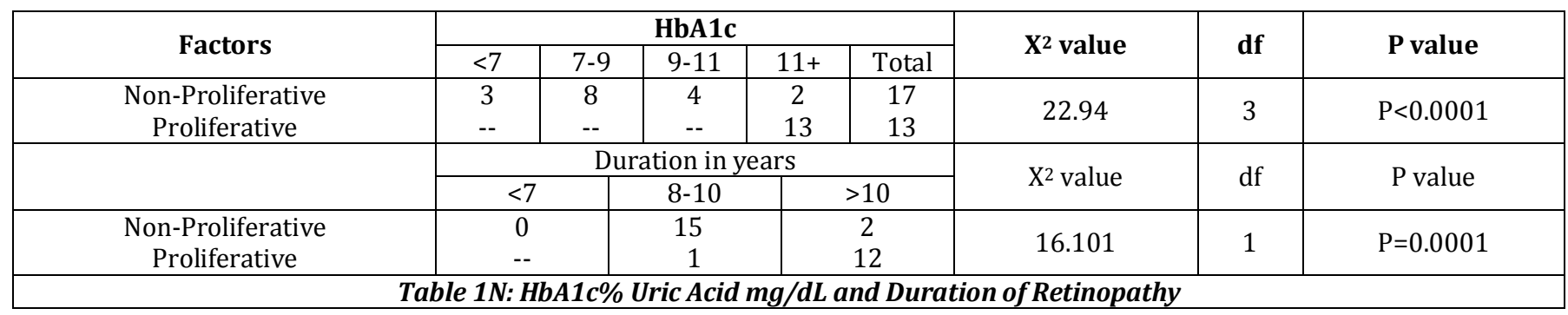

\begin{tabular}{|c|c|c|c|c|c|c|c|c|}
\hline \multirow{2}{*}{ Factors } & \multicolumn{5}{|c|}{ Uric Acid Level (mg/dL) } & \multirow{2}{*}{$X^{2}$ value } & \multirow{2}{*}{ df } & \multirow{2}{*}{$P$ value } \\
\hline & $<2$ & $2-4$ & $4-6$ & $6+$ & Total & & & \\
\hline Non-Proliferative & -- & 14 & 3 & -- & 17 & & & \\
\hline Proliferative & -- & 12 & 1 & -- & 13 & 0.0640 & 1 & 0.8003 \\
\hline
\end{tabular}

\section{DISCUSSION}

Diabetes mellitus is a group of metabolic disease characterized by hyperglycaemia resulting from a defect in insulin secretion, insulin action or both. Diabetic retinopathy is an ocular complication of diabetes mellitus and is one of the leading causes of blindness in industrial countries. ${ }^{4}$ The current study, "A study of glycated haemoglobin and serum uric acid in proliferative and non-proliferative diabetic retinopathy" was undertaken in an effort to understand the interplay between glycated haemoglobin and serum uric acid level and their role in the mechanisms leading to diabetic retinopathy.

The test Group I comprised of patients with controlled diabetes and without retinopathy. Most of the patients belonging to this group visited the OPD for correction of refractive error or removal of foreign body from the cornea, stye, chalazion, etc.

The test Group II comprised of patients with uncontrolled diabetes mellitus with retinopathy. The patients belonging to this group attended the OPD for vision disturbance and were referred to the Retina Clinic for further evaluation. This group also included patients admitted for cataract surgery and intraocular lens implantation. Majority of the patients belonging to this group presented with hyperglycaemia due to irregular treatment.

The pathogenic link between hyperglycaemia and complication of diabetes is attributed to oxidative stress, which plays a primary role. ${ }^{5}$ However, investigators still discuss whether oxidative stress precedes the appearance of complications or whether it merely reflects the presence of complications. ${ }^{6}$
In the present study, it was observed that the controlled diabetes cases in test Group I had fasting glucose values below $126 \mathrm{mg} / \mathrm{dL}$ and HbA1c values below.7 In test Group II, cases presenting with retinopathy had fasting blood glucose values above $120 \mathrm{mg} / \mathrm{dL}$ and HbA1c values above. ${ }^{7}$ The test Group II, which was further sub-classified into nonproliferative and proliferative retinopathy showed $\mathrm{HbA1c}$ values above $6 \%$ and $11 \%$ respectively, which tally with another study. 7 The serum uric acid values in both nonproliferative and proliferative retinopathy were below 4 $\mathrm{mg} / \mathrm{dL}$. (Table $1 \mathrm{~K}$ ), A study reported low serum uric acid in diabetics with complications. ${ }^{8}$

\section{AGE}

From the present study, it is seen that majority of the patients are in the age group of 50-59 years, followed by 60-69 years as shown in Table 1B, which tally with a study. ${ }^{9}$

\section{Fasting Serum Glucose}

In the present study, it is seen that the fasting serum glucose values in test Group I subjects are between $77-126 \mathrm{mg} / \mathrm{dL}$. There was significant elevation $(\mathrm{P}<0.0001)$ of mean fasting glucose value in the test Group I patients as compared to the mean fasting glucose value of $76.66 \pm 7.18 \mathrm{mg} / \mathrm{dL}$ of the control group. There was also significant difference $(\mathrm{P}<0.0001)$ in the mean serum fasting glucose level of the test Group II and test Group I patients with a mean fasting glucose value of $238.93 \pm 73.86 \mathrm{mg} / \mathrm{dL}$. Thus the diabetic status of test Group II, controlled diabetic status of test Group I and normal 
glycaemic status of the control group are established and all the groups are suitable for the proposed study.

In the present study, it is seen that the mean value of HbA1c of $10.73 \pm 2.810$ in test Group II was significantly raised $(\mathrm{P}<0.0001)$ when compared to the normal control group and test Group I with HbA1c levels of $4.90 \pm 0.813$ and $5.03 \pm 1.060$ respectively. However, there was no significant difference $(\mathrm{P}=0.596)$ between the mean value of $\mathrm{HbA1c}$, between control group and test Group I, reflecting a relation between glycaemic control and glycated haemoglobin level in blood. These results are in accordance with similar type of previous studies by (Nur Kebapci et al, 1999).

\section{Serum Uric Acid Levels}

In the present study, the mean serum uric acid values of test Group II subjects $2.99 \pm 0.605$ as compared to control group $4.136 \pm 0.901$ and test Group I $4.346 \pm 0.942$ is significantly lowered $(\mathrm{P}<0.0001)$ (Table 1-I). However, no significant difference $(\mathrm{P}=0.381)$ is observed in the mean serum uric acid values of control and test Group I subjects. These results are in accordance with the previous study, which reported lower uric acid levels in type 2 diabetes, particularly in complicated patients with peripheral neuropathy, which may be due to oxidative stress that decreases the antioxidant capacity of the body involving uric acid. ${ }^{8}$ When correlated the $\mathrm{HbA} 1 \mathrm{c} \%$ with serum uric acid in test Group II, there was no significant correlation observed with $\mathrm{P}=.2937$

\section{Relation between HbA1c\% Duration and Uric Acid levels with Type of Retinopathy}

Relation between $\mathrm{HbA} 1 \mathrm{c} \%$, duration and serum uric acid with retinopathy was assessed by chi-square test. In the present study, majority of patients with non-proliferative diabetic retinopathy had $\mathrm{HbA} 1 \mathrm{c} \%$ between $7-9 \%$ and in proliferative stage above $11 \%$, which is highly significant $(\mathrm{P}<0.0001)$ (Table $1 \mathrm{~L}$ ).

This is in accordance with DCCT study, which showed that total lifetime exposure to glycaemia was the principal determinant of the risk of retinopathy and there was a continuous though non-linear relationship between this risk and the mean level of HbA1c.

\section{Duration}

Majority of patients with non-proliferative retinopathy had duration of diabetes mellitus below 10 years and most of the patients with proliferative retinopathy had a duration of more than 10 years. The relation between retinopathy and duration is significant $(\mathrm{P}=0.0001)$. This is in accordance with study of (Klein R et al, 1984).

\section{Relation of Serum Uric Acid with Retinopathy}

From the present study, it is seen that the serum uric acid levels in majority of the cases of non-proliferative and proliferative retinopathy are below $4 \mathrm{mg} / \mathrm{dL}$, and the relation between serum uric acid level and retinopathy is not significant with $\left(\mathrm{P}=\right.$.8003). This tally with a study. ${ }^{10}$

However, the exact nature of how glycation of haemoglobin and serum uric acid are related to complication of diabetic retinopathy requires further studies in this population with various study designs.

\section{CONCLUSION}

Diabetic retinopathy remains a prevalent complication of diabetes mellitus. Current modalities either aim to prevent progression of diabetic retinopathy with annual ocular examinations and glycaemic and blood pressure control or are geared towards preserving functional vision of high risk patients with photocoagulation and vitrectomy. However, an insight into the biochemical details of the disease process will better help health care providers to understand and treat diabetic retinopathy.

The molecular mechanisms of hyperglycaemia induced endothelial dysfunction are not fully understood, but oxidative stress clearly plays a key role in the initial insult.

Multiple biochemical pathways known to increase the production of reactive oxygen species have been linked to hyperglycaemia/diabetes induced vascular injury including glucose autoxidation, polyol pathway and formation of Advanced Glycation End Products (AGE). ${ }^{11}$ These processes have been suggested to be initiated by hyperglycaemia induced superoxide production in the mitochondrial electron transport chain. (Nishikawa T et al, 2000).

HbA1c is a valuable index of hyperglycaemia and protein glycation of the previous 3-4 months. ${ }^{12}$ It is also known to be a source of free radicals by reducing molecular oxygen. ${ }^{13}$ The United Kingdom Prospective Diabetes Study Group (UKPDS) has found that each 1\% decrease in HbA1c levels are associated with a $37 \%$ reduction in retinopathy in type 2 diabetes. As a large number of patients who develop microvascular complications such as retinopathy have little or no symptoms until late stages of the disease, aggressive research is being warranted in order to better understand the intricate details that connect hyperglycaemia with diabetic retinopathy.

In test Group II patients, the HbA1c levels were increased $(\mathrm{P}<0.0001)$ as compared to test Group I and control group. The serum uric acid levels were lowered in test Group II $(\mathrm{P}<0.0001)$, in comparison to test Group I and control group indicating oxidative stress, which decreases the antioxidant capacity of the body involving uric acid, conforming to the findings of other authors.

Therefore, screening of diabetic patients is essential for early detection, prompt treatment and planned regular follow-up by good comprehensive ocular examination and biochemical tests. Besides, research assessing the role of new pharmacologic agents which metabolically affect retinopathy and sizeable clinical trials focused on the long-term benefits of these drugs will help achieve the goal of decreasing the incidence of blindness in diabetic patients.

The current study "A study of Glycated Haemoglobin and Serum Uric Acid in proliferative and non-proliferative diabetic retinopathy" was undertaken to understand the role of hyperglycaemia in the mechanism leading to chronic complications of diabetes mellitus.

\section{REFERENCES}

1. Manaviat MR, Afkhami M, Shoja MR. Retinopathy and microalbuminuria in type IIdiabetic patients. BMC ophthalmol 2004;4:9-12.

2. Tapp RJ, Shaw JE, Harper CA, et al. The prevalence of the factors associated with diabetic retinopathy in the Australian population. Diabetics care 2003;26(6):1731-7. 
3. Carl A Burtis, Edward R Ashwood, David E Burns. Tietz textbook of clinical chemistry and molecular diagnostics. Elsevier New Delhi 2008; $4^{\text {th }}$ ed:879-86.

4. Kahn HA, Hiller R. Blindness caused by diabetic retinopathy. Am J ophthalmol 1974;78(1):58-67.

5. Baynes JW. Role of oxidative stress in development of complications in diabetes. Diabetes 1991;40(4):405-12.

6. Thorpe SR, Baynes JW. Role of oxidative stress in diabetic complications: a new perspective on old paradigm. Diabetes 1999;48(1):1-9.

7. Klein R, Klein BE, Moss SE. The Wisconsin epidemiologic study of diabetic retinopathy, II: prevalence and high risk of diabetic retinopathy when age at diagnosis is less than 30 years. Arch Ophthalmol 1984;102(4):520-6.

8. Isan Hamo M. Serum uric acid concentrations in patients with type 2 diabetes mellitus during diet or glibenclamide therapy. Pak J Med Sci 2007;23(3):361-5.
9. Nur Kebapci Y, Belgin Efe, Fahrettin Akyuh, et al. Oxidative stress and anti-oxidant therapy in type- 2 diabetes mellitus. Turkish Journal of Endocrinology and Metabolism 1999;4:153-62.

10. Feldman T, Weitzman S, Biedner B. Retinopathy and serum uric acid in diabetics. Harefuah 1995;128(11):6813,744 .

11. Giugliano D, Ceriello A, Paolisso G. Oxidative stress and diabetic vascular complications. Diabetes care 1996;19(3):257-67.

12. Kennedy L, Baynes JW. Non enzymatic glycosylation andthe chronic complications of diabetes: overview. Diabetologia 1994;26:93-8.

13. Satoh $M$, Bessho $T$, Imaizumi $K$, et al. Increased erythrocyte aggregation in diabetes mellitus and its relationship to glycosylated haemoglobin and retinopathy. Diabetologia 1984;27(5):517-21. 